\title{
Stimulus segmentation in the visual search task
}

\author{
CESAR GALERA \\ Universidade de São Paulo, São Paulo, Brazil \\ EDERALDO JOSÉ LOPES \\ Universidade Federal de Uberlândia, Uberlândia, Brazil \\ and \\ MICHAEL VON GRÜNAU \\ Concordia University, Montreal, Quebec, Canada
}

\begin{abstract}
Four experiments were conducted in order to study the segmentation process in a visual search task with relevant stimuli (target and distractors) randomly distributed among textural elements. The basic idea was that a parallel segmentation process of the relevant stimuli would contribute to the overall reaction time independently of the contribution of the number of relevant stimuli. In the first experiment, with relevant stimuli and textural elements that differed in the orientation of their component lines, texture presence interacted with number of relevant stimuli and with target presence. These results were not favorable to the parallel segmentation hypothesis. In the second and third experiments, in which the relevant and the textural stimuli differed in orientation and in the luminance contrast of their component lines, the results support a parallel segmentation process for the higher contrast conditions. In these experiments, the effect of texture presence was greater on target-absent than on target-present trials. Experiment 4 shows that the search can be restricted to the high-contrast relevant stimuli when the number of these stimuli is constant and the number of textural stimuli changes from trial to trial. The present results suggest that the relevant stimuli can be segmented in parallel and then submitted to a restricted analysis, even when they are scattered among textural stimuli.
\end{abstract}

In the real world, we often search for objects or scenes that are placed among useless, irrelevant elements. These background elements typically can be grouped and segregated, allowing an efficient search for a specific target. The importance of grouping for the performance of the visual search task has been largely recognized. In the first version of the feature integration theory (FIT), Treisman and Gelade (1980) showed that the detection of a target that shared its features with the distractors could be achieved through a serial scan of groups of stimuli, provided that the field contained groups of spatially adjacent stimuli. In this earlier version of the FIT, neither perceptual grouping nor parallel detection of visual features required focal attention. Treisman (1982) suggested that preattentive grouping creates separate feature maps that can be analyzed in parallel but that this is possible only when the stimuli are spatially grouped.

Partial results of this study were presented at the XXVI International Congress of Psychology, Montreal, August 1996. This research was supported by Grants CNPq/522624/95 and FAPESP/1997/6347-8 to C.G. and by NSERC Grant 047 to M.v.G. We thank Jeremy Wolfe, Lester Krueger, and two anonymous reviewers for very useful comments on the first version of this paper. Correspondence concerning this article should be addressed to C. Galera, DPE FFCLRP-USP, Av. Bandeirantes 3900, Ribeirão Preto-SP, 14040-901, Brazil (e-mail: algalera@) usp.br).
Later work by Egeth, Virzi, and Garbart (1984) and also by Nakayama and Silverman (1986) suggested that visual search could be restricted to a set of stimuli with a common feature, such as color or depth plane, despite their distribution in the field. The stimulus selection would enable the allocation of attention to a restricted subset, with a performance gain when the target is defined by a conjunction of features present in the distractors. Treisman and Sato (1990) found a strong correlation between performances in a segregation task and in a search task, suggesting that stimuli that are easily segregated are easily searched. The segregation process might occur through the inhibition of some sets of stimuli, leaving other sets available for attentional processing. Other models also proposed that the search is achieved through the recursive organization of groups of stimuli (Grossberg, Mingolla, \& Ross, 1994; Humphreys \& Müller, 1993).

The importance of the grouping process was also suggested in the search surface model proposed by Duncan and Humphreys (1989). In this model, the first stage of processing is a perceptual description in which the parts that belong to the same whole are linked together. This grouping process occurs in parallel and without resource limitations. The search is difficult when the target is more similar to the distractors and when these are dissimilar among themselves; in these cases, the target will be grouped with the distractors, and a first segmentation of the relevant stimuli will not isolate the target from them. 
The need of a new segmentation in smaller areas will increase the time needed for target detection. On the other hand, when the target and the distractors differ substantially and the distractors are homogeneous and group strongly, the target will be easily segmented.

Perceptual grouping does not play an important role in the guided search (GS) model (Wolfe, 1994; Wolfe, Cave, \& Franzel, 1989). In this model, attention is directed to single items that cause more activity in the activation map. The activation map acts as the weighted sum of the activations produced in the feature maps, allowing attention to be directed to its more active locations. For example, activities of the red and vertical features of a target, among green vertical and red horizontal distractors, will sum up in the activation map. The summed activities of these features can be used to guide the attention to the most promising locations of the activation map. The attention is allocated in a serial way from the greatest to the smallest activation location in the activation map. Although attention cannot be directed to more than one single item at a time, there is an implicit grouping in the GS model when similar items have similar activations (Wolfe, 1994). The study of Friedman-Hill and Wolfe (1995) suggests, indeed, that the attentional process can be restricted to only one subset of items, ignoring the remaining ones.

\section{THE PRESENT STUDY}

The studies on selection of stimuli in visual search have shown that the search for the target can be restricted to a subset of elements presented in the visual field (see also Kaptein, Theeuwes, \& van der Heijden, 1995; Poisson \& Wilkinson, 1992; Theeuwes, 1996; Zohary \& Hochstein, 1989). In the present study, we investigated the nature of the segmentation process that enables a group of stimuli to be analyzed separately by a limited-capacity attentional process. Is this segmentation process a parallel or a serial one? We investigated this issue in a visual search task with relevant stimuli (target and distractors) randomly distributed among textural elements, from which they differed in the orientation and luminance of their line components. The basic assumption was that the relevant stimuli would be preattentively segregated from the textural elements and that the output of this segregation process, the grouped relevant stimuli, would be submitted to a focal attention analysis.

To test this idea, we applied the additive factor logic (Sternberg, 1969; see also Egeth \& Dagenbach, 1991; Pashler \& Badgio, 1985). According to this logic, experimental factors that influence different stages of processing will have additive effects on reaction time (RT), whereas factors that act on the same stage of processing will interact. In our study, we will consider two factors: presence of textural elements and number of relevant stimuli $\left(N_{\mathrm{rs}}\right)$ in the visual field. If the presence of texture and the $N_{\mathrm{rs}}$ act on different stages of processing, they will have independent effects on RT. Otherwise, a serial segmentation process will be revealed by a superadditive interaction between the effects of the texture and the $N_{\text {rs }}$.

Most studies consider that segmentation or grouping of stimuli in the visual search task occurs in parallel in an early processing stage and that the analysis of the segmented stimuli occurs in a later processing stage. The studies carried out by Beck (1972) suggest that the stimuli are segregated early through differences in properties that are discriminable when attention is distributed over the whole stimulus field. Stimuli that differ in orientation, brightness, or size of their lines are segregated more easily than stimuli that differ in the way their features are combined. It seems that the visual system makes simultaneous discriminations of stimulus differences and that these differences enable stimulus grouping before the focusing of attention on smaller or more restricted stimulus field areas.

In this study, our subjects performed a visual search task with its relevant stimuli (target and distractors) distributed among textural stimuli. Relevant and textural stimuli were letter-like Ts that differed in their orientation and in the luminance of their component lines. We asked whether the segmentation of the relevant stimuli could be performed in parallel. The results of Experiment 1 suggest that relevant and textural stimuli differing in orientation of their component lines are not segmented in parallel. The results obtained in Experiments 2 and 3 present some evidence that the segmentation of the relevant stimuli could be performed in parallel, provided that the luminance contrast between these stimuli is great enough. The results of Experiment 4 suggest that the search for the target can be restricted to the relevant stimuli only in the higher contrast condition.

\section{EXPERIMENT 1}

In Experiment 1, the stimuli relevant to the visual search task were Ts with vertical and horizontal segments, and the textural stimuli were Ts with $45^{\circ}$ slanted segments. Line orientation is segregated preattentively (Beck, 1972, 1982), and we expected that vertical and horizontal Ts would group together and would segregate easily from the textural stimuli whose component lines were oriented at $45^{\circ}$. If so, the time taken for a parallel segregation of the relevant stimuli would be independent of the time taken to analyze the segregated stimuli.

\section{Method}

Subjects. Twelve undergraduate students from the Universidade de São Paulo at Ribeirão Preto, 6 male and 6 female, were paid to take part in this experiment. All the subjects reported having normal or corrected-to-normal vision.

Stimuli and Apparatus. The stimuli were presented on a 15 -in. color monitor (Philips 15b) with a $60-\mathrm{Hz}$ frame rate. The distance from the screen to the subject's eye was about $70 \mathrm{~cm}$. The stimuli were luminous Ts composed of segments $7 \mathrm{~mm}$ in length and $0.9 \mathrm{~mm}$ in width $\left(0.57^{\circ} \times 0.07^{\circ}\right.$ of visual angle $)$, presented on a dark background. The distractors were Ts in the vertical position, the target 


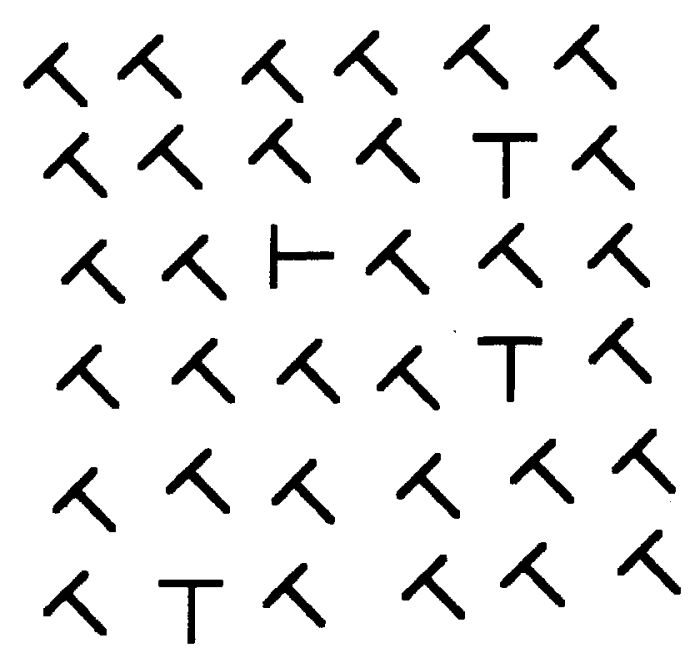

Figure 1. Example of stimulus display used in Experiment 1: textural elements (slanted Ts), target (horizontal T), and distractors (vertical Ts). The actual stimuli were white on black.

was a $\mathrm{T}$ rotated $90^{\circ}$ to the left, and the textural stimuli were Ts rotated $45^{\circ}$ to the left. The relevant and textural stimuli were presented in bright white $\left(68.9 \mathrm{~cd} / \mathrm{m}^{2}\right)$ on a black background $\left(0.02 \mathrm{~cd} / \mathrm{m}^{2}\right)$. The luminance of the stimulus segments was calibrated by means of a Tektronix photometer, model $\mathrm{J} 17$ with a $\mathrm{J} 1803$ head. The relevant stimuli, target and distractors, were randomly distributed on a matrix with $6 \times 6$ cells in a $9 \times 9 \mathrm{~cm}$ area on the monitor screen $\left(7.4^{\circ} \times 7.4^{\circ}\right.$ of visual angle). The textural stimuli occupied the remaining free cells of the matrix (Figure 1). The position of each stimulus in the matrix cell was slightly displaced from column and row center, in order to minimize configuration patterns. The target was present on $50 \%$ of the trials, replacing one of the distractors.

Procedure. Each subject took part in an experimental session divided into three blocks of 168 trials each. Each treatment resulting from the combination of three factors $-N_{\mathrm{rs}}$ (two, four, and six), target (present, absent), and texture (present, absent) - was randomly presented 14 times in each block of trials $(3 \times 2 \times 2 \times 14=168)$.

The first trial in the block was preceded by a signal (*) in the center of the screen. This signal was on for $500 \mathrm{msec}$ and was replaced by the stimuli of the first trial. The stimuli remained on the screen until the subject's response. The response was immediately followed by a feedback signal, "+" (correct response) or "-" (incorrect response). This signal remained on the screen for $1,800 \mathrm{msec}$, until the beginning of the next trial. In each trial, the subject had to press the $F$ of the keyboard if the target was present and the $J$ if the target was absent. The first 10 trials of each block, the trials with wrong responses, and the trials with RTs less than $200 \mathrm{msec}$ or more than $3,000 \mathrm{msec}$ were presented again in the same block of trials and were not taken into account for the analysis of the results. At the beginning of the session, the subjects were informed that textural stimuli would be presented at random in half of the trials. The subjects were also told that the textural stimuli were not relevant to their task and that they should pay attention only to the relevant stimuli. The subjects were asked to respond without errors and as fast as possible. Mean RT was based only on correct trials.

\section{Results and Discussion}

Figure 2 shows the mean correct RT for each experimental condition. Mean RTs obtained by each subject in the different treatments were submitted to an analysis of variance (ANOVA) with repeated measures, with $N_{\text {rs }}$, tar- get, and texture presence as factors. Mean RT increased significantly as a function of the $N_{\mathrm{rs}}$ in the field $[F(2,22)=$ $81.06, p<.0001]$ and as a function of the presence of the texture $[F(1,11)=344.96, p<.0001]$ and was greater for the target-absent trials $[F(1,11)=113.02, p<.0001]$. The effect of the textural stimuli on RT was greater when there were more relevant stimuli in the field $[F(2,22)=$ $3.99, p=.033]$ and for the target-absent trials $[F(1,11)=$ $217.95, p=.0001]$. Mean RT increased with the same slope for target-present and target-absent trials. This is confirmed by the nonsignificant interaction between the $N_{\text {rs }}$ and target presence $[F(2,22)<1]$.

The slope of the RT function obtained in the trials without textural stimuli was greater than the 6-7 msec/item sometimes suggested as characteristic of parallel processing (Humphreys, Quinlan, \& Riddoch, 1989; Treisman \& Souther, 1985), but it was too flat to be considered as an indication of a serial search process. A slope of around $13 \mathrm{msec} /$ item has been associated with a search strategy in which groups of items are checked in parallel (Treisman \& Gormican, 1988), with a search process restricted to a subset of selected items (Egeth et al., 1984), or with a GS strategy (Wolfe, 1994). The equality of the slopes for target-present and target-absent trials obtained in the texture-absent trials also suggests that the small $N_{\text {rs }}$ used in this experiment could be analyzed in parallel with a capacity-limited parallel process (Pashler, 1987).

The significant effect of the textural stimuli on the slope of the RT function, confirmed by the interaction between the $N_{\mathrm{rs}}$ and the presence of texture, suggests that the segmentation process was dependent on the number of stimuli to be segmented. These results suggest that the segregation of the relevant stimuli was not conducted simultaneously for all the relevant stimuli in the field or that the system cannot analyze smaller areas inside segregated groups of stimuli. It seems that the orientation difference alone was not sufficient for the parallel segmentation of the relevant stimuli or that the segmented group did not last a sufficiently long time to be analyzed, which, in turn, required the segmentation of new groups.

The presence of the texture affected both the intercept and the slope of the RT function. The effect was stronger for the intercept of the RT function for the target-absent trials $(326 \mathrm{msec})$ than for the intercept of the target-present trials $(85 \mathrm{msec})$. This interaction between the presence of the target and of the textural stimuli contradicts the hypothesis that the subjects first segregated the relevant stimuli and then looked for the target, as is proposed by some models (Grossberg et al., 1994; Zohary \& Hochstein, 1989). If this was the case, the effect of the textural stimuli on the intercept should be the same for target-present and target-absent trials.

The error rate of each subject in all the treatments was submitted to the same ANOVA as that for RT. The error rate increased with the $N_{\mathrm{rs}}[F(1,11)=15.11, p=.003]$. There were more misses than false alarms $[F(1,11)=10.4$, $p=.008]$, and there were more errors in the texture-present trials than in the texture-absent trials $[F(1,11)=12.31$, 

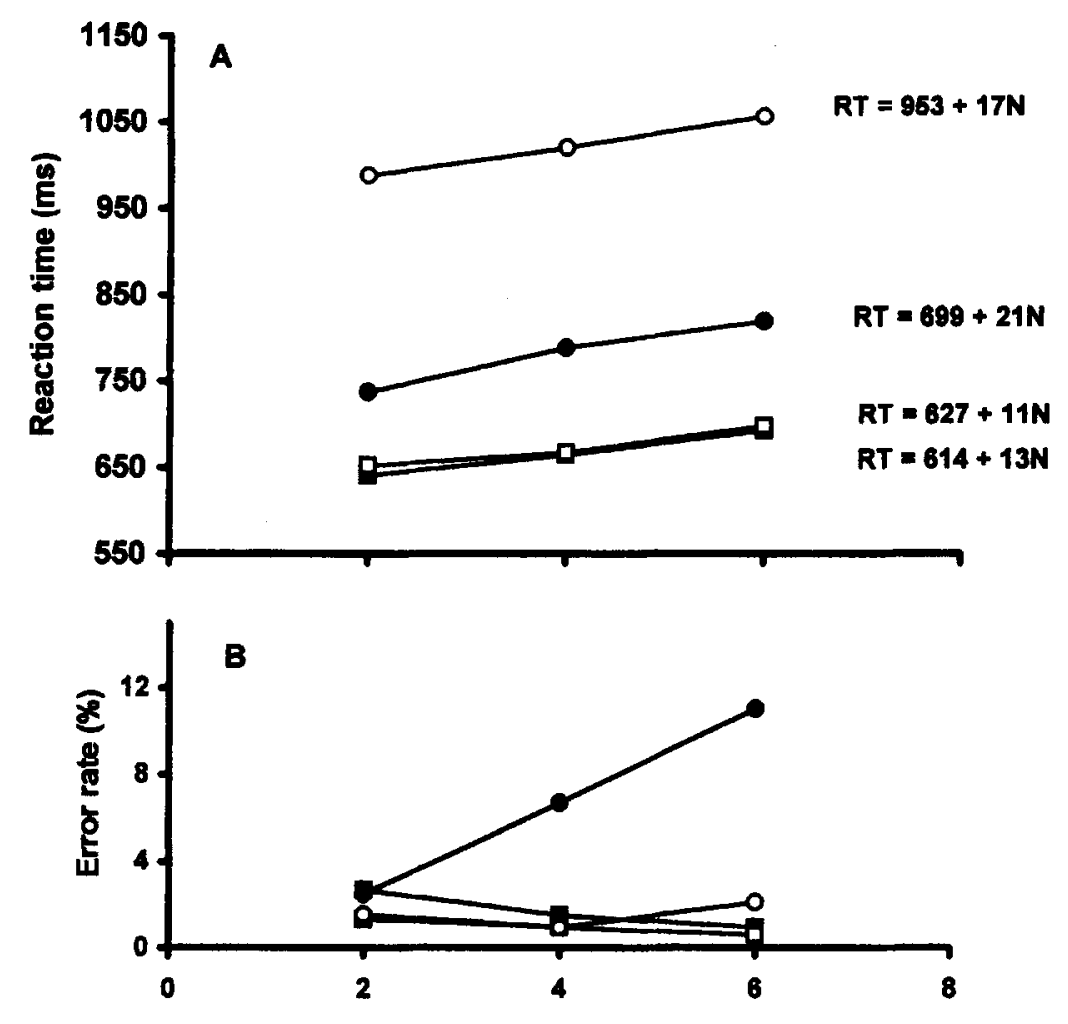

Number of relevant stimull

Figure 2. Experiment 1: Mean reaction times (RTs) and error rates for trials with a texture (circles), without a texture (squares), with a target (filled symbols), and without a target (empty symbols). The RT functions are based on least-squares fits.

$p=.005]$. In the texture-present trials, error rate tended to increase with increasing $N_{\text {rs }}$, and this effect was practically restricted to target-present trials, as is confirmed by the interaction between the presence of texture and of target $[F(1,11)=6.93, p=.023]$. In the texture-absent trials, the error rate tended to decrease with increasing $N_{\mathrm{rs}}$, suggesting a speed-accuracy tradeoff. To test the significance of this tendency, we calculated, for each subject, the correlation between RT and misses and that between RT and false alarms, across the three levels of $N_{\text {rs }}$ in textureabsent trials. One-sample $t$ tests showed that neither the mean correlation between misses and RT $[t(11)=-1.14$, $p=.28]$ nor the mean correlation between false alarms and RT $[t(11)=-.99, p=.343]$ differed significantly from zero.

The proportion of misses, responding target absent in target-present trials, increased sharply as a function of $N_{\mathrm{rs}}$ in texture-present trials. According to Krueger (1984), the preponderance of misses could be explained by the focused rechecking and by premature termination factors. In texture-absent trials, the spuriously perceived target can be singled out and rechecked as the only different stimulus in the field. It is possible to consider that the presence of textural elements in the field acts as a noise in the decision system, increasing the number of spurious differ- ences to be rechecked or making the recheck more difficult. If there is some noise in the decision system, more differences should be examined in order to prevent a miss. If the subject rechecks only a few differences, the probability of a miss will increase with the number of relevant stimuli in texture-present trials. According to the supposition of premature termination, the subject may prematurely respond target absent before examining all the stimuli in the field, increasing the proportion of misses in displays with greater $N_{\mathrm{rs}} \mathrm{s}$. Together, the lesser focused rechecking and the greater premature termination could explain the relatively faster RT and higher errors on the texture- and target-present trials than on the texture- and target-absent trials.

The effect of texture presence in this experiment resembles that obtained by Egeth, Folk, and Mullin (1989, presented in Folk \& Egeth, 1989, Figure 1) in a samedifferent task with relevant stimuli (vertical and horizontal line segments) distributed among textural elements. Despite the differences in stimuli and tasks used in both experiments, the presence of texture had a strong effect on the slope of the RT function and interacted (our results) or tended to interact (results of Folk \& Egeth, 1989) on the intercept with target presence/difference. According to Folk and Egeth, the decrement in performance 

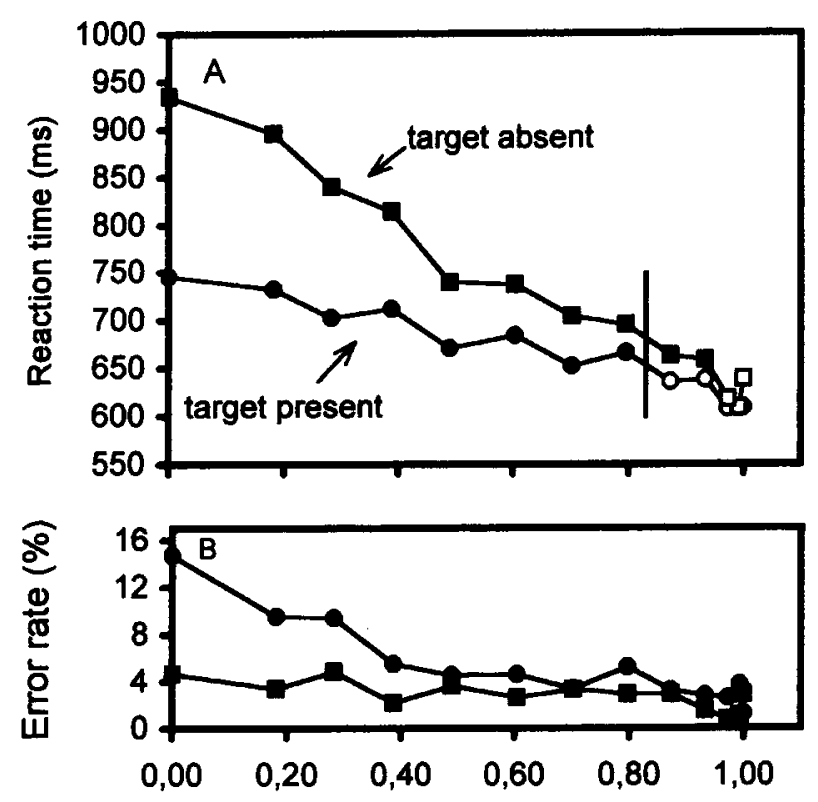

Michelson contrast

Figure 3. Experiment 2: Mean reaction times and error rates as a function of the Michelson contrast between relevant and textural stimuli. Open symbols represent means that do not differ from means obtained without textural elements in the field (two points on the extreme right). There is no significant difference between means for target-present and target-absent trials to the right of the vertical line.

of the same-different task obtained when textural elements were present could be attributed to a decision factor. These authors consider that the presence of texture elements creates a noise in the same-different decision process. This noise could change the criterion response with the $N_{\text {rs }}$ present in the field, or it could change the search strategy.

In summary, our results contradict the hypothesis that the segmentation of the relevant stimuli occurs in parallel. The orientation difference alone does not seem to be sufficient for a parallel segmentation process; that is, the segmentation process based on differences in orientation depends on the number of stimuli to be segmented. Zohary and Hochstein (1989) also suggested that orientation (when compared with color) is not the most dominant dimension for the parallel figure-ground segregation process.

\section{EXPERIMENT 2}

The results of Experiment 1 have shown that, in a visual search task, the segregation of the relevant stimuli that differ from textural elements only in the orientation of their line components requires serial processing. Similar results were found in other studies (Galera, 1997) and suggest that a greater difference between relevant and textural stimuli would be necessary for a parallel segmentation process. The results of Beck (1967) showed that, besides the orientation of line segments, the relative brightness of stimuli is an important factor for the segregation process. Ts in the canonical position and Ts slanted to $45^{\circ}$ are perceived as two groups when one of the patterns is brighter than the other. In this experiment, we aimed to establish the luminance difference between textural and relevant stimuli that would permit a parallel segmentation process. Six bright relevant $T$ stimuli were distributed among textural elements, from which they differed in the orientation and luminance of their component lines.

\section{Method}

Subjects. Seven volunteers, graduate and undergraduate students from the Universidade de São Paulo at Ribeirão Preto, plus one of the authors (C.G.), participated in two sessions of about $30 \mathrm{~min}$. All the subjects reported having normal or corrected-tonormal vision.

Stimuli and Apparatus. The stimuli and apparatus were the same as those in the last experiment. The relevant stimuli were presented with a luminance level of $68.9 \mathrm{~cd} / \mathrm{m}^{2}$. The textural stimuli had a different orientation than the relevant stimuli and were presented at different gray levels, ranging from bright white to dark gray $(68.9,47.7,38.6,30.4,23.6,17.0,12.0,7.8,4.7,2.4,0.95$, and $\left.0.24 \mathrm{~cd} / \mathrm{m}^{2}\right)$. With these values, the Michelson contrast $[(\mathrm{H}-\mathrm{L}) /$ $(\mathrm{H}+\mathrm{L})]$ between the luminance of relevant $(\mathrm{H})$ and textural $(\mathrm{L})$ stimuli ranged from 0 to .99 . In each trial, the visual display consisted of 30 textural elements, with the same luminance level, randomly distributed on a $6 \times 6$ virtual matrix $(9 \times 9 \mathrm{~cm})$; the relevant stimuli occupied the other six positions in the matrix. In half of the visual displays, the target replaced one distractor.

Procedure. The luminance level of the textural elements varied from trial to trial. In the first session, the textural elements were presented at six contrast ratios $(0, .18, .39, .6, .8$, and .93$)$ or were absent (contrast ratio of 1 ). In the second session, the textural elements were presented with the remaining contrast ratios $(.28, .49$, $.7, .87, .97$, and .99 ) and were always present. The session was divided into four trial blocks. In each block, there were 16 trials at each luminance level (including the absence of texture as a luminance level). In this way, there were 448 trials in one session and 384 in the other. The luminance level and the target presence varied from trial to trial. The subjects were informed that the textural stimuli were not relevant to their task and that they should pay attention only to the relevant stimuli. The subject's task was to press the $F$ key when the target was present and the $J$ key when the target was absent. A feedback ("+" or "-") was presented in the screen center immediately after the subject's response and stayed on as a fixation point until the beginning of the next trial, $1,500 \mathrm{msec}$ after the response.

\section{Results and Discussion}

Mean RT was affected by the luminance of the textural elements $[F(11,77)=19.78, p<.0001]$, by the presence of the target $[F(1,7)=15.90, p=.005]$, and by the interaction between these factors $[F(11,77)=10.42, p<$ $.0001]$. In the target-present trials, the RT was not affected by the presence of textural elements for the Michelson contrasts larger than or equal to $.87[t(7)=1.66, p=.142]$. In the target-absent trials, there were no effects of texture after a contrast ratio of $.97[t(7)=0.88, p=.409]$. The differential effect of texture luminance on RT in trials with and without targets is an important diagnostic of processing strategy. There was no difference between the RTs for target-present and target-absent trials beyond a contrast of $.87[t(7)=1.82, p=.112$; points to the right of the ver- 
tical line in Figure 3]. For lower values (points to the left of the vertical line), the effect of luminance contrast was greater for trials without a target.

The error rate was submitted to the same ANOVA as that for RT. As in the first experiment, there were more misses $(4.96 \%)$ than false alarms $[2.81 \% ; F(1,7)=6.9$, $p=.034]$. The proportion of misses decreased as a function of Michelson contrast $[F(12,84)=3.08, p=.001]$. The false alarm proportion was not affected by the change in luminance $[F(12,84)=1.12, p=.355]$.

The obtained equality of the mean RTs for trials with and without targets at high contrast levels can be considered as evidence that the search may have been exhaustive or that the relevant stimuli may have been analyzed in parallel, as was suggested by Pashler (1987). For smaller luminance differences, the presence of the textural elements makes the task difficult, but more difficult in the trials without a target. We suggest that, at high contrast levels, the equality of the RTs for trials with and without a target and the absence of a texture effect on RT might be considered as evidence for parallel processes of target detection and textural segmentation. But, at low contrast ratios, the segmentation process becomes more difficult, perhaps changing to a serial one, with changes in the group size of the segmented relevant stimuli.

Nagy and Sanchez (1992) also showed that, in a visual search task with a fixed number of distractors, RT decreased as a function of luminance difference between target and distractors, up to an asymptotic level. According to these authors, the results suggest that there was a change in the task strategy around a critical value for the difference in luminance between target and distractors. The search was serial for luminance differences smaller than a critical value and parallel when the difference was larger. Pins and Bonnet (1996) showed that, in some choice RT tasks, the effect of stimulus luminance is independent of the effect of other factors on RT. This independence is explained in terms of the successive processing of stimulus intensity and other dimensions of the task. At a very early level of neural processing, the first available information concerns the intensity of the visual stimulus; the processing of other dimensions can only begin when intensity processing has come to an end. In our experiment, the effect of luminance contrast on RT was independent of target presence only for the higher contrast levels. Thus, in these trials only, stimulus intensity may have been processed in parallel, before other dimensions could be analyzed.

The results obtained in this exploratory experiment showed that RT was not affected by the presence of a texture if the textural elements and relevant stimuli had a contrast above .97 for trials without a target and above .87 for trials with a target. This value should not be considered as an all-or-none threshold. The processes underlying the gradual change presented in Figure 3 should be still better determined. For our immediate purpose, it is suf- ficient to know that, for contrast ratios smaller than this value, the RTs for target-present and target-absent trials were affected differentially by the presence of textural elements. In consequence, the luminance values to be used in the next experiment were chosen around this point.

\section{EXPERIMENT 3}

In this experiment, we studied stimulus segmentation in a visual search task with a variable $N_{\text {rs }}$ randomly distributed among textural elements. The relevant and textural stimuli differed in orientation and luminance of their line segments. The basic idea is that the relevant stimuli will be preattentively segmented when the difference between the luminance of the textural elements and that of the relevant stimuli is sufficiently large. An early segregation process that occurs simultaneously over all the stimuli in the field ought to contribute to the RT independently of the serial analysis effect; that is, the effect of the presence of the textural elements ought to be independent of the effect of the $N_{\mathrm{rs}}$.

\section{Method}

Subjects. Fifteen subjects, 7 males and 8 females, all undergraduate students from the Universidade de São Paulo at Ribeirão Preto, were paid to participate in two experimental sessions, one lasting about $1 \mathrm{~h}$ and the other lasting about $30 \mathrm{~min}$. All the subjects reported having normal or corrected-to-normal vision.

Stimuli and Apparatus. The same apparatus and stimuli as those in the last experiment were used here. The difference was that the textural elements were presented at only three luminance levels, $4.7,12.0$, and $23.6 \mathrm{~cd} / \mathrm{m}^{2}$. The relevant stimuli were presented, as before, at $68.9 \mathrm{~cd} / \mathrm{m}^{2}$, which yielded three contrast ratios $(.87, .7$, and .49) between the luminance levels of the relevant and the textural stimuli.

Procedure. The experiment was divided into three conditions, one for each contrast ratio. In each condition, the 12 treatments resulting from the combination of the $N_{\mathrm{rs}}$ (two, four, six), target (present, absent), and texture (present, absent) were presented 42 times ( $42 \times$ $12=504$ ). Each condition was divided into three blocks of 168 trials $(3 \times 168=504)$. All the subjects passed through all the treatments in two experimental sessions. One session could be composed of one or two conditions. For 8 subjects, the first session had only one condition; 7 subjects worked on two conditions in the first session. The order of luminance levels was counterbalanced across subjects. The presence of target, the presence of texture, and the number of stimuli in the field varied randomly from trial to trial. The other details were the same as those in the previous experiments.

\section{Results and Discussion}

Figure 4 presents a summary of the mean RTs for four factors: contrast between the luminance of relevant and textural elements $(.49, .7, .87)$, texture (present, absent), target (present, absent), and $N_{\text {rs }}$ (two, four, and six).

Mean RT for texture-present and texture-absent trials were submitted to separate ANOVAs. In trials without texture, RT was affected only by the $N_{\mathrm{rs}}[F(2,28)=87.68$, $p<.0001]$. Mean RT increased as a function of the $N_{\mathrm{rs}}$ with a slope of $11.8 \mathrm{msec} /$ item $\left(r^{2}=.996\right)$. No other main 

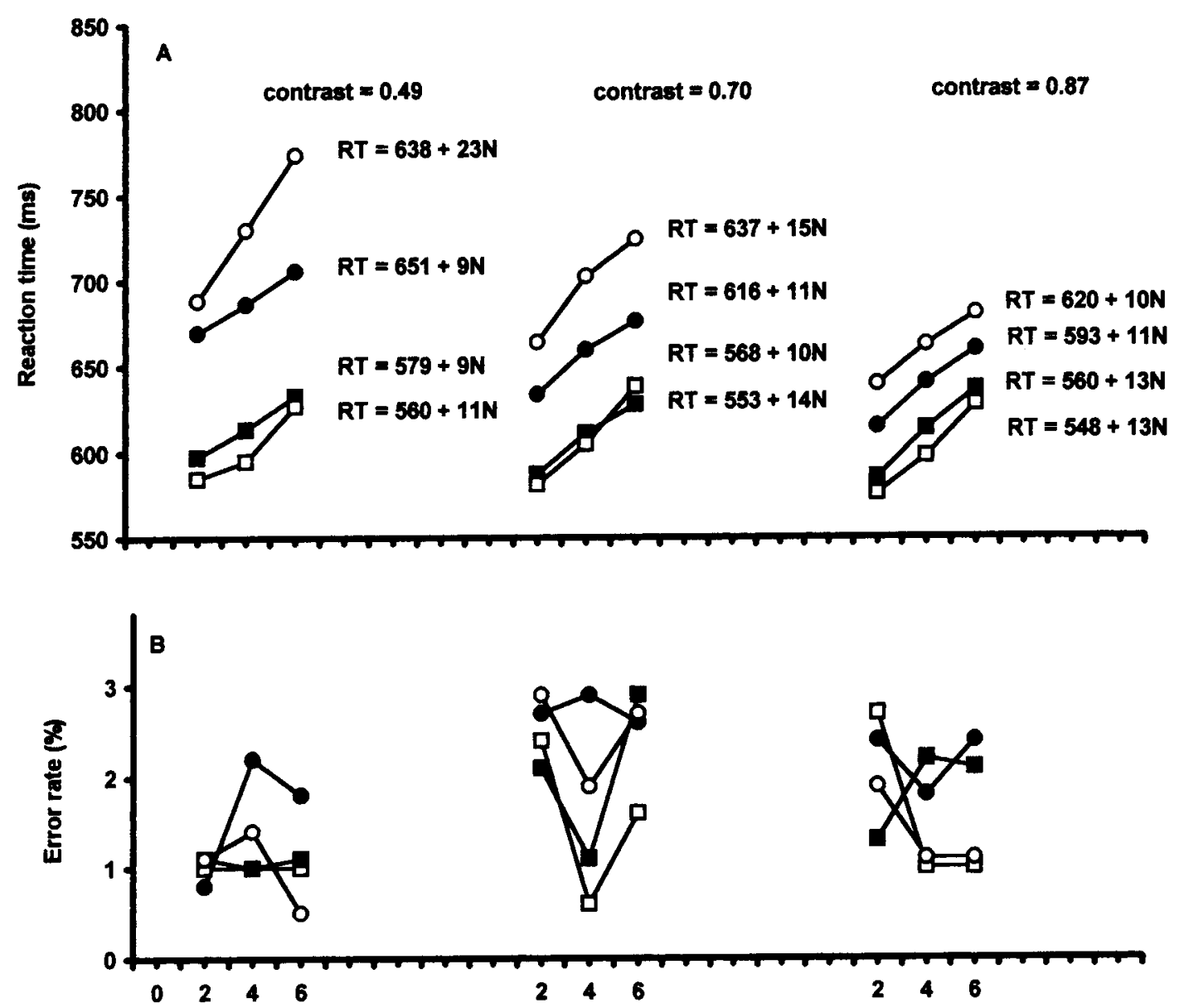

Number of relevant stimuli

Figure 4. Experiment 3: Mean reaction times (RTs) and error rates for trials with texture (circles) and without texture (squares), with a target (filled symbols), and without a target (open symbols). The RT functions are based on least-squares fits.

factor or interaction had a significant effect on RT on these trials. No experimental factor had a significant effect on the error rates.

In trials with texture, mean RT increased with the $N_{\text {rs }}$ $[F(2,28)=58.85, p<.0001]$, was greater on targetabsent trials $[F(1,14)=10.89, p=.005]$, and decreased for the higher contrast level $[F(2,28)=7.68, p=.002]$. The increase of RT as a function of $N_{\mathrm{rs}}$ was greater for the target-absent trials $[F(2,28)=3.74, p<.036]$. The effect of contrast was also greater in target-absent trials $[F(2,28)=4.04, p=.028]$. These factors, $N_{\mathrm{rs}}$ and the contrast between relevant and textural stimuli, had independent effects on RT $[F(4,56)=1.29, p=.282]$ but established a significant three-way interaction with target presence $[F(4,56)=4.43, p<.004]$. This interaction can be better observed in Figure 4. Contrast level affected the intercept of the RT functions for target-present trials but not their slopes. In target-present trials, the slope of the mean RT function was about $10.3 \mathrm{msec} /$ item $\left(r^{2}=.996\right)$.
In target-absent trials, the slope of the RT function varied from $21.5 \mathrm{msec} /$ item $\left(r^{2}=1.00\right)$ in the low-contrast condition (.49) to $10.3 \mathrm{msec} /$ item $\left(r^{2}=.997\right)$ in the high-contrast condition (.87). In the intermediate-contrast condition $(0.70)$, the slope of the target-absent RT function was $15 \mathrm{msec} /$ item $\left(r^{2}=.998\right)$. No experimental factor had a significant effect on the error rates.

According to the logic of this study, if the effect of $N_{\mathrm{rs}}$ on RT does not depend significantly on texture presence, that would indicate parallel segmentation of the relevant stimuli. To evaluate the combined effect of the presence of textural elements and $N_{\mathrm{rs}}$ on RT, we performed a separate ANOVA in each contrast condition. This analysis shows that the slope of the RT function (effect of the $N_{\mathrm{rs}}$ ) does not depend on the presence of textural elements in the high-contrast condition $[F(2,28)=1.65, p=.21]$ and in the intermediate-contrast condition $[F(2,28)=0.63, p=$ .539]. Only in the low-contrast condition was the interaction between presence of texture and $N_{\mathrm{rs}}$ significant 
$[F(2,28)=4.06, p=.028]$. In all the contrast conditions, the texture presence interacted significantly with the target presence (all $p s<.032$ or less).

In the low-contrast condition, we obtained some of the results obtained in the first experiment, except that the presence of texture changed the slope of the RT function for the target-absent trials, but not for the target-present trials. This differential effect suggests that the presence of texture introduced a change in the search strategy and that this change was more pronounced in the target-absent trials. Also, the effect of texture in the target-present trials suggests a parallel segmentation process of the relevant stimuli.

The independence between the effects of texture and of $N_{\mathrm{rs}}$ on RT, obtained in the higher contrast conditions, is in accordance with a model of parallel segmentation, in which the relevant stimuli would be segmented simultaneously all across the stimulus field. The time cost of this segmentation, around $30 \mathrm{msec}$ in target-present and $70 \mathrm{msec}$ in target-absent trials, can be seen at the intercept of the RT function. We are assuming that, when the textural elements are present, the first segmentation will segregate the relevant stimuli from the textural elements, and a second segmentation will be necessary to segregate the target from the distractors. The interaction between the presence of the target and the contrast level, however, poses a problem for a serial model in which segmentation is followed by the detection of the target. The contrast between the luminance of relevant and of textural stimuli had a greater effect on RT in trials without the target. This differential effect suggests that more processing is needed to reach a no-target response. According to Pashler and Badgio (1985), the interaction between visual quality and target presence might be considered as evidence that these factors act on stages related to decision processes. Our results suggest that the stage affected by the presence of texture and target can be an early perceptual process, in which segmentation takes place. It is possible that the segmentation of relevant stimuli and target detection might be occurring at the same time. The target is not necessarily detected after the segregation; it might be detected while the relevant stimuli are being segregated.

Why did the texture have this different effect on targetpresent and target-absent trials? When the effect of the presence of textural elements was independent of $N_{\mathrm{rs}}$ but interacted with target presence, as in the higher contrast level conditions (.70 and .87), it is possible that the selection of relevant stimuli and target detection were, at least in part, simultaneous. But when the presence of textural elements affected the slope of the target-absent trials, it is possible that the different effects of textural luminance on target-present and target-absent trials may be due to the later processes of the search. Pashler and Badgio (1985) also found it difficult to explain why an early process, such as the segmentation process seems to be, should be more affected when the target was not present. According to these authors, the visual quality of the stimuli could affect the rate at which evidence for stimulus identification is accumulated. Therefore, if the subject had different criteria for target-absent and target-present trials, an interaction between perceptual and decisional factors would be expected.

Folk and Egeth (1989) also suggested that the presence of textural stimuli in a same-different task could introduce some noise into the decision process. They argued that the presence of texture increases the time needed for the decision process to reach a correct response. According to Krueger (1984), the lower RT function in the textureand target-present trials, relative to texture- and targetabsent trials, could be explained, at least in part, to lesser rechecking and greater premature termination on those trials, besides the possibility of target-present responses being self-terminating.

In summary, the effect of texture depended on the value of the contrast ratio and on target presence. In the targetpresent trials, the effect of texture on RT was independent of the effect of $N_{\mathrm{rs}}$. In the target-absent trials, the presence of texture tended to interact with $N_{\mathrm{rs}}$ as the difference between the luminance of relevant and of texture element stimuli became smaller. The results showed that there is a change in the processing strategies as a function of contrast ratio. The slope ratios varied from 1.1:1 to $2.3: 1$, as a consequence of the stronger effect of luminance on the slopes of the target-absent trials; the slopes of the targetpresent trials remained stable, despite changes in the contrast ratio.

\section{EXPERIMENT 4}

It is possible that the effect of texture presence on the slope of the RT function for target-absent trials and on the low-contrast condition of Experiment 3 could be explained by a serial segmentation process. It is also reasonable to consider the possibility of a serial (re)check of some textural elements, as was proposed by Folk and Egeth (1989) and by Krueger (1984). In the higher contrast conditions, where texture affected only the intercept of the RT function, we think of this increase as being the time needed for the perceptual segregation of the relevant stimuli. But it is also reasonable to think that this increase in the intercept of the RT function could be attributed to the analysis of a roughly constant number of texture elements. If a few of the textural stimuli were submitted to some attentional analysis, the time this takes could be added to mean RT, independent of the $N_{\mathrm{rs}}$. In Experiment 4, we investigated whether search is limited to the relevant stimuli in the high-contrast condition. The $N_{\mathrm{rs}}$ was fixed, and the number of textural stimuli $\left(N_{\mathrm{ts}}\right)$ was varied. If the subjects were to search for the target only among the relevant segregated relevant stimuli, it would be expected that the search time would be independent of the $N_{\mathrm{ts}}$.

\section{Method}

Subjects. Seven subjects, 5 undergraduate students from the Universidade de São Paulo at Ribeirão Preto, 3 male and 2 female, 

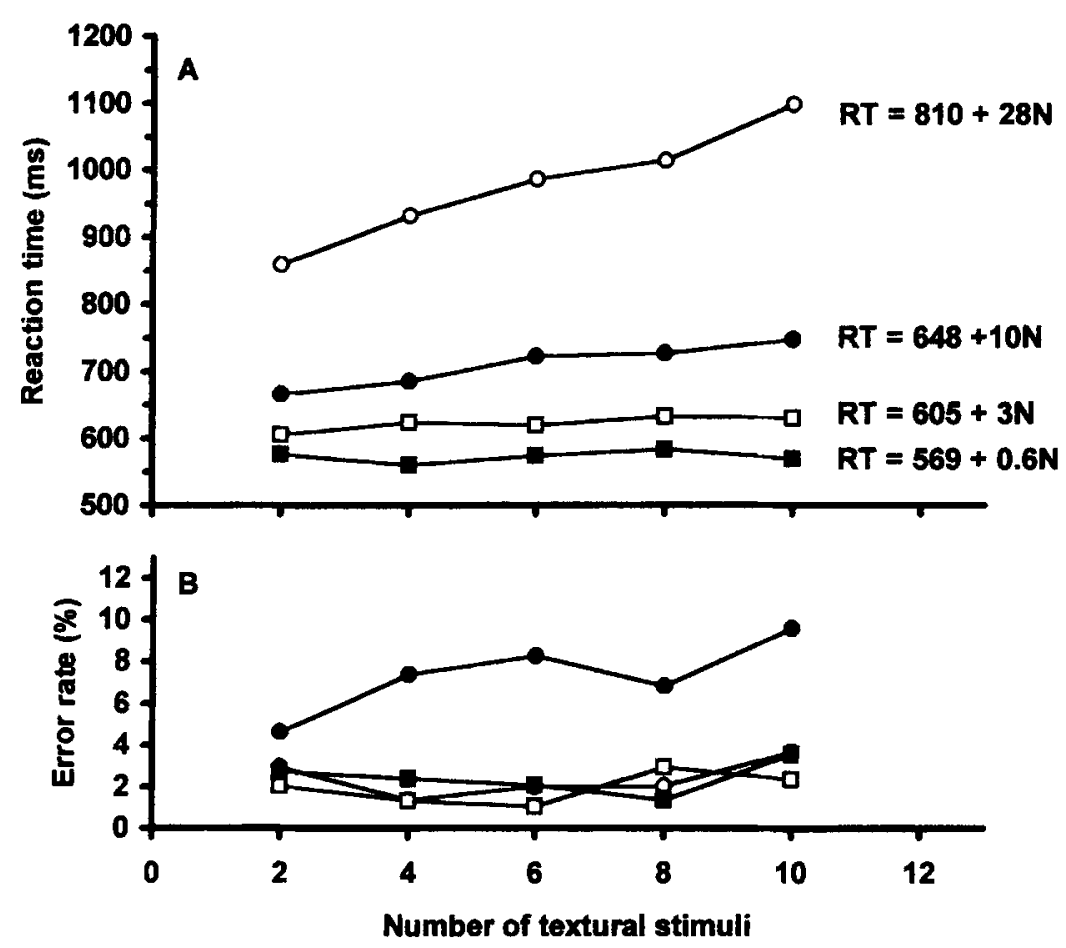

Figure 5. Experiment 4: Mean reaction times (RTs) as a function of the number of textural stimuli for high-contrast (squares) and low-contrast (circles) conditions, in trials with a target (filled symbols) and without a target (open symbols). The RT functions are based on least-squares fits.

plus 2 authors (C.G. and E.J.L.), participated in one experimental session lasting about $1 \mathrm{~h}$. The students were recruitud and were paid to participate. All the subjects reported having normal or correctedto-normal vision. Data were lost for an additional, 8th subject, owing to a hardware malfunction

Stimuli and Apparatus. The same apparatus and stimuli as those in the previous experiments were used here. The target (horizontal T) and the distractors (vertical Ts) were presented at $59.8 \mathrm{~cd} / \mathrm{m}^{2}$. The textural stimuli, Ts rotated $225^{\circ}$ from the vertical position, were presented at $3.4 \mathrm{~cd} / \mathrm{m}^{2}$ and at $59.8 \mathrm{~cd} / \mathrm{m}^{2}$, which yielded Michelson contrast ratios of .89 and 0 , respectively, between the luminance levels of the relevant and the textural stimuli. The stimuli were presented on a black background and were distributed on a $6 \times 6$ matrix $\left(8^{\circ} \times 8^{\circ}\right)$.

Procedure. The $N_{\text {ts }}$ was kept constant at 6 , and the $N_{\mathrm{ts}}$ was varied from 2 to 10 . The experimental session was divided into two parts. The contrast between relevant and textural stimuli was high (.89) in one part and low (0) in the other part. Each part was divided into four blocks of 100 trials each. The 10 treatments that resulted from the combination of two factors $-N_{\mathrm{ts}}(2,4,6,8$, and 10) and target (present, absent)-were randomly presented 10 times in each block of trials $(10 \times 5 \times 2=100)$. Four subjects were presented first with four blocks of high-contrast stimuli and then with four blocks of low-contrast stimuli; the other 4 subjects were tested in the inverse order. The other details were the same as those in the previous experiments.

\section{Results and Discussion}

Figure 5 presents a summary of the mean RTs. Mean RTs obtained in the low- $(0)$ and high-contrast (.89) conditions were submitted to separate ANOVAs with repeated measures in two main factors: $N_{\mathrm{ts}}(2,4,6,8$, or 10$)$ and target (present, absent).

In the low-contrast condition ( 0$)$, mean RT increased as a function of $N_{\mathrm{ts}}[F(4,24)=17.14, p<.0001]$ and was larger in the target-absent trials $[F(1,6)=23.88, p=.003]$. The interaction between these factors, evident in the differences between the slopes for target-present and targetabsent trials, was also significant $[F(4,24)=5.39, p=.003]$. The slopes of $27.9 \mathrm{msec} /$ item $\left(r^{2}=.98\right)$ for target-absent trials and of $10.2 \mathrm{msec} /$ item $\left(r^{2}=.95\right)$ for target-present trials suggest, first of all, that in the low-contrast condition, the search was not limited to the six relevant stimuli present in the field. Second, the slope ratio between target absence and target presence of 2.74:1 suggests that some rechecking was needed before a response could be selected.

The mean error rate for the low-contrast condition was submitted to the same analysis as that for RT. The error rate tended to be higher for target-present trials $(7.32 \%)$ than for target-absent trials $(2.39 \%)$, but this factor just failed to reach a significant level $[F(1,6)=5.84, p=.052]$. Neither $N_{\mathrm{ts}}[F(4,24)=1.21, p=.333]$ nor the interaction between this and target presence $[F(4,24)<1]$ was significant.

In the high-contrast condition (.89), RT was not affected by $N_{\mathrm{ts}}[F(4,24)=1.99, p=.128]$ or by target presence $[F(1,6)=4.40, p=.081]$. None of the slopes for the RT functions $-2.9 \mathrm{msec} /$ item for target-absent trials and 
$0.6 \mathrm{msec} /$ item for target-present trials - was significantly different from zero $[t(6)<1.64]$. This finding is consistent with the supposition that the search was restricted only to the relevant stimuli. Overall, there was a $2.2 \%$ error rate in the high-contrast condition. No experimental factor had a significant effect on error rate.

Recent results obtained by Theeuwes (1996), with stimuli defined by color and orientation, show that search can be performed in parallel only when the target is placed in a spatially contiguous subset of distractors. Our results show that search could be restricted only to the relevant stimuli, even when they are distributed among other stimuli in the display, provided the contrast luminance was great enough.

\section{GENERAL DISCUSSION}

In this study, we investigated the nature of stimulus segmentation in a visual search task with relevant stimuli (target and distractors) randomly distributed among textural elements. The relevant and textural stimuli differed in the orientation and luminance of their line components; the target and the distractors differed in the way their line components were combined. The basic idea was that a segmentation process acting simultaneously on all the stimuli in the field would output a group of similar stimuli that could be analyzed by an attentional process. The parallel segregation of the relevant stimuli would be evidenced by the independent effects of textural elements and $N_{\text {rs }}$ on RT. On the other hand, a superadditive interaction between $N_{\text {rs }}$ and texture would be expected when the segregation of the relevant stimuli was achieved by a serial process.

The results obtained in the high-contrast condition of Experiment 3 show that RT was affected independently by the presence of texture and by $N_{\mathrm{rs}}$. The increase in RT in the texture-present trials of this condition can be considered an estimation of the time needed to select the relevant stimuli. In this condition, the presence of the textural elements does not seem to change the search strategy. The equality of the slopes and of the ratios between slopes for target-present and target-absent trials obtained with and without texture suggests that, in the texture trials, the relevant stimuli were selected and submitted to an analysis similar to that performed in the texture-absent trials. Other studies also found evidence that, in a conjunction search, a subset of distractors can be selected in parallel for later analysis (e.g., Poisson \& Wilkinson, 1992; Zohary \& Hochstein, 1989). Bravo and Blake (1990) reached similar conclusions in a study that used a visual search task with textured displays. In their study, the stimuli were textured patterns that differed from the background texture in the nrientation of their line segments. The time needed to detect the textured target was faster when the background texture was not present, but the slopes obtained with and without texture background were similar. The authors attributed this difference in absolute RT to the time needed for the segmentation of the stimuli from the background, which would require a constant amount of time, independent of the number of stimuli to be segmented.

The interactions between $N_{\text {rs }}$ and the presence of texture in the first experiment and in the low-contrast condition of Experiment 3 suggest that orientation differences alone were not sufficient for parallel segmentation of the relevant stimuli. Zohary and Hochstein (1989) found that color, and not orientation, seems to be the dominant dimension for stimulus selection. Egeth et al. (1984) also found that search was more efficient when subjects attended to color than when they attended to form. In our study, the luminance difference between the relevant and the textural stimuli is the dominant feature for the selection process. This is suggested in the last three experiments. In Experiment 2, the increase in the contrast ratio between the luminance of the relevant and of the textural stimuli was followed by a general reduction in RT and by a change of the search strategy. For a contrast ratio of about .87 , the effect of texture was the same for target-present and target-absent trials. In Experiment 3, the slope of the RT function for the high-contrast texture-present trials was the same as that for the texture-absent trials. In Experiment 4, RT did not change as a function of $N_{\mathrm{ts}}$ when the contrast luminance was high. All these cases suggest that luminance contrast is an efficient selection criterion when the relevant stimuli are distributed among other elements.

The interaction between the presence of texture and of target suggests that the effect of texture was not limited to an early perceptual analysis. In all the experiments, the effect of texture was consistently greater on target-absent trials than on target-present trials. Pashler and Badgio (1985) suggest that perceptual factors such as the visual quality of stimuli could affect the rate at which the evidence for one response or another increases. Folk and Egeth (1989) suggest that the interaction between texture presence and target presence might be due to noise or to a change introduced by texture in a decision process. Another suggestion that the texture could also have affected a decision process comes from the increasing proportion of misses that occurred in the low-contrast conditions of Experiments 1, 2, and 4. A rechecking strategy, as was suggested by Krueger (1984), or a more strict criterion to respond target absent, as was suggested by Chun and Wolfe (1996), could explain the greater effect of texture on the RT of target-absent trials and on the preponderance of misses.

At a first glance, the independent effect of texture and $N_{\mathrm{rs}}$ and the larger effect of texture on the intercept of the RT functions for target-absent trials might suggest a sequential segmentation process (Grossberg et al.,1994; Theeuwes, 1996). The first segmentation step would separate the relevant from the textural stimuli; a second step could segment the target, if it is present. Otherwise, a recursive segmentation on smaller field areas will enable a target-absent response. But, it is also possible that the effect of texture presence could be smaller in target-present 
trials. The segmentation of the relevant stimuli and target detection could be achieved, at least in part, simultaneously. The overlapping between relevant stimulus segmentation and target detection might occur in conditions in which the detection of the target is fast, as in the present experiments.

Friedman-Hill and Wolfe (1995) studied the selection process with an experimental paradigm that required subjects to search for a target of odd orientation among stimuli of a prespecified color. The target was a red line oriented $X^{\mathrm{o}}$; distractors were green lines oriented $X^{\mathrm{o}}$ and red lines oriented $Y^{\circ} . X$ and $Y$ changed from trial to trial. Beforehand, the subjects were aware only of the target's color, not of its orientation. According to the authors, the orientation information could be useful only after the red subset had been selected. Their results showed that the slopes of the RT function obtained in the subset search are smaller than the slopes of standard serial search, and this was considered evidence that the search could be restricted to the red subset. In the subset search, RT was 200-300 msec slower than the RT obtained in a control conjunction search, and this increase in time was attributed to the selection of the red items. This substantial increase in RT could also be attributed to target identification (the subjects did not know the target's orientation). Our results showed that, when the subjects knew beforehand the target's identity, the selection of relevant stimuli could take a smaller amount of time, around $30-70 \mathrm{msec}$.

Many other studies have suggested that visual search can be restricted to a subset of stimuli (e.g., Egeth et al., 1984; Kaptein et al., 1995; Nakayama \& Silverman, 1986). McLeod, Driver, Dienes, and Crisp (1991) found that, if subjects can segment the display into two groups of stimuli, they can pay attention to only one of the groups, with a performance gain. Our results show that the grouping is not an all-or-none process. Provided that textural and relevant stimuli are different enough, the grouping process may be achieved independently of the $N_{\mathrm{rs}}$. The independence between the presence of texture and $N_{\mathrm{rs}}$ does not mean, however, that the segmentation process always needs to be completed before the beginning of the analysis of the relevant stimuli. It is also necessary to take into account the interaction between the presence of texture and of target. This interaction is a difficult one. It is possible to say, on the one hand, that the effect of texture is smaller on target-present trials, and we suggest that this effect could be considered as evidence that the segmentation of the relevant stimuli and target detection overlap in time. It is also possible to say, on the other hand, that the effect of texture is greater on target-absent trials, and this suggests that the presence of texture is accompanied by a change in search strategy on these trials, perhaps affecting the criteria that determine a target-absent response.

\section{REFERENCES}

BEck, J. (1967). Perceptual grouping produced by line figures. Perception \& Psychophysics, 2, 491-495.
BECK, J. (1972). Similarity grouping and peripheral discriminability under uncertainty. American Journal of Psychology, 85, 1-19.

BECK, J. (1982). Texture segregation. In J. Beck (Ed.), Organization and representation in perception (pp. 285-317). Hillsdale, NJ: Erlbaum.

Bravo, M., \& Blake, R. (1990). Preattentive vision and perceptual grouping. Perception, 19, 515-522.

Chun, M. M., \& Wolfe, J. M. (1996). Just say no: How are visual searches terminated when there is no target present? Cognitive Psychology, 30, 39-78.

DunCaN, J., \& Humphreys, G. W. (1989). Visual search and stimulus similarity. Psychological Review, 96, 433-458.

EgeTh, H., \& DaGenbaCH, D. (1991). Parallel versus serial processing in visual search: Further evidence from subadditive effects of visual quality. Journal of Experimental Psychology: Human Perception \& Performance, 17, 551-560.

EGeth, H., Virzi, R. A., \& Garbart, H. (1984). Searching for conjunctively defined targets. Journal of Experimental Psychology: Human Perception \& Performance, 10, 32-39.

FOLK, C. L., \& EGETH, H. (1989). Does the identification of simple features require serial processing? Journal of Experimental Psychology: Human Perception \& Performance, 15, 97-110.

FRIEDMAN-Hill, S., \& Wolfe, J. (1995). Second-order parallel processing: Visual search for the odd item in a sub-set. Journal of Experimental Psychology: Human Perception \& Performance, 21, 531 551

GALERA, C. (1997). Agrupamento par similaridade e busca visual [Similarity grouping and visual search]. Psicologia: Teoria \& Pesquisa, 13, 261-268

Grossberg, S., Mingolla, E., \& Ross, W. D. (1994). A neural theory of attentive visual search: Interaction of boundary, surface, spatial, and object representation. Psychological Review, 101, 470-489.

HUMPHREYS, G. W., \& MüLlER, H. (1993). Search via recursive rejection (SERR): A connectionist model of visual search. Cognitive Psychology, 25, 43-110.

Humphreys, G. W., Quinlan, P. T., \& Riddoch, M. J. (1989). Grouping processes in visual search: Effects with single- and combinedfeature targets. Journal of Experimental Psychology: General, 118, 258-279.

Kaptein, N. A., Theeuwes, J., \& van der Heijden, A. H. C. (1995). Search for a conjunctively defined target can be selectively limited to a color-defined subset of elements. Journal of Experimental Psychology: Human Perception \& Performance, 21, 1053-1069.

KRUEGER, L. E. (1984). Self-termination in same-different judgments: Multiletter comparison with simultaneous and sequential presentation. Journal of Experimental Psychology: Learning, Memory, \& Cognition, 10, 271-284

MCLeod, P., Driver, J., Dienes, Z., \& Crisp, J. (1991). Filtering by movement in visual search. Journal of Experimental Psychology: Human Perception \& Performance, 17, 55-64.

NAGY, A. L., \& SANCHEZ, R. R. (1992). Chromaticity and luminance as coding dimensions in visual search. Human Factors, 34, 601-614.

Nakayama, K., \& Silverman, G. H. (1986). Serial and parallel processing of visual feature conjunctions. Nature, 320, 264-265.

Pashler, H. (1987). Detecting conjunctions of color and form: Reassessing the serial search hypothesis. Perception \& Psychophysics, 41, 191-201.

Pashler, H., \& Badgio, P. C. (1985). Visual attention and stimulus identification. Journal of Experimental Psychology: Human Perception \& Performance, 11, 105-121.

Pins, D., \& BonNeT, C. (1996). On the relation between stimulus intensity and processing time: Piéron's law and choice reaction time. Perception \& Psychophysics, 58, 390-400.

Poisson, M. E., \& Wilkinson, F. (1992). Distractor ratio and grouping process in visual conjunction search. Perception, 21, 21-38.

STERNBERG, S. (1969). The discovery of processing stages: Extensions of Donders' method. In W. G. Koster (Ed.), Attention and performance II (pp. 276-315). Amsterdam: North-Holland.

THEEUWES, J. ( I996). Parallel search for a conjunction of color and orientation: The effect of spatial proximity. Acta Psychologica, 94, 291307. 
Treisman, A. (1982). Perceptual grouping and attention in visual search for features and for objects. Journal of Experimental Psychology: Human Perception \& Performance, 8, 194-214.

Treisman, A., \& Gelade, G. (1980). A feature integration theory of attention. Cognitive Psychology, 12, 97-136.

Treisman, A., \& GormiCan, S. (1988). Feature analysis in early vision: Evidence from search asymmetries. Psychological Review, 95, 15-48. Treisman, A., \& Sato, S. (1990). Conjunction search revisited. Journal of Experimental Psychology: Human Perception \& Performance, 16, 459-478.

Treisman, A., \& Souther, J. (1985). Search asymmetry: A diagnostic for preattentive processing of separable features. Journal of Experimental Psychology: General, 114, 285-310.
WoLfe, J. M. (1994). Guided Search 2.0: A revised model of visual search. Psychonomic Bulletin \& Review, 1, 202-238.

Wolfe, J. M., Cave, K. R., \& Franzel, S. L. (1989). Guided Search: An alternative to the feature integration model for visual search. Journal of Experimental Psychology: Human Perception \& Performance, $15,419-433$.

ZOHARY, E., \& HoChSTEIN, S. (1989). How serial is serial processing in vision? Perception, 18, 191-200.

(Manuscript received November 20, 1997; revision accepted for publication October 19, 1998.) 